\title{
Numerical Bifurcation Analysis of Double +1 Multiplier in $\mathbb{Z}_{3}$-Symmetric Maps: A Case Study in the Cournot Triopoly Game Model
}

\author{
Reza Mazrooei-Sebdani ${ }^{a}$, , Zohreh Eskandari ${ }^{a \dagger}{ }^{\dagger}$ and Hil G.E. Meijer ${ }^{b} \ddagger$ \\ ${ }^{a}$ Department of Mathematical Sciences, Isfahan University of Technology, Isfahan 84156-83111, Iran. \\ ${ }^{b}$ Department of Applied Mathematics, University of Twente, 7500 AE Enschede, The Netherlands.
}

September 7, 2018

\begin{abstract}
We consider a resonance 1:1 bifurcation with $\mathbb{Z}_{3}$-symmetry in a discrete-time dynamical system. We employ standard normalization and center manifold reduction techniques. With this, we obtain the normal form up to cubic order and also explicit formulas for the critical normal form coefficients. We provide an implementation of our algorithm that can be used in the numerical bifurcation toolbox MATCONTM. We illustrate our analysis with a Cournot triopoly model from economics.
\end{abstract}

Keywords: Resonance $1: 1$ (Double +1 ) Bifurcation, $\mathbb{Z}_{3}$-Symmetry, Map, Critical Normal Form Coefficient, Numerical Continuation.

\section{Introduction}

Symmetric bifurcation theory has a rich history with many beautiful theoretical results, see e.g. [7, 8], and also see, in particular for maps, [3]. In many case studies, the form of the

${ }^{*}$ Corresponding author. E-mail addresses: mazrooei@cc.iut.ac.ir(R. Mazrooei-Sebdani)

${ }^{\dagger}$ E-mail address: Zohreh.eskandari@math.iut.ac.ir (Z. Eskandari)

${ }^{\ddagger}$ E-mail address: h.g.e.meijer@utwente.nl (H.G.E. Meijer) 
model allows an analytical exploration of the effect of symmetry. Meanwhile, the development of numerical tools to study bifurcations with symmetry is limited.

There has been much more progress in a numerical approaches for generic dynamical systems. This has culminated in the packages AUTO07P 2 and MATCOnT[4]. There is a special version MATCONTM[10] that is devoted to maps. This toolbox supports numerical continuation and bifurcation analysis of fixed points and cycles of iterated maps. MATCONTM detects limit point, period doubling and Neimark-Sacker points and supports continuation of these bifurcations in two control parameters. Along such bifurcation curves, all codimension 2 bifurcations are also detected. The critical normal form coefficients of codim 1 and codim 2 bifurcations are computed as developed in [13. These coefficients are needed to verify non-degeneracy and to determine the corresponding bifurcation scenario.

In the presence of symmetries, certain generic conditions in the analysis are no longer satisfied. Therefore, we studied how to adapt MATCONTM in the case of symmetries. Here we report on a case study of a symmetric bifurcation in maps. In particular, we focus on algorithms and changes in the toolbox MATCONTM needed to support the bifurcation analysis in a numerical context.

For concreteness, we consider a family of smooth discrete-time dynamical systems

$$
x \rightarrow f(x ; \alpha), \quad x \in \mathbb{R}^{n} ; \quad \alpha \in \mathbb{R}^{2} .
$$

We consider a codimension 2 bifurcation where $x_{0}$ is a fixed point, i.e. $f\left(x_{0}\right)=x_{0}$, such that the linearization $A=f_{x}\left(x_{0}, 0\right)$ has a double multiplier +1 . In this study, we also assume that $f$ has $\mathbb{Z}_{3}$-symmetry, and that the bifurcation occurs w.r.t. the $\mathbb{Z}_{3}$-symmetry. So the symmetry has a nontrivial cyclic action on the state variables, but a trivial action on the parameters. We present a detailed theoretical and numerical analysis of this bifurcation. The truncated normal form up to third order on the center manifold for this case is given by ([12], Chapter 9, Section 9.5.4)

$$
\mathbf{z} \mapsto\left(\left(1+\alpha_{1}\right)+i \alpha_{2}\right) \mathbf{z}+\tilde{a}(\alpha) \overline{\mathbf{z}}^{2}+(c(\alpha)+i d(\alpha)) \mathbf{z}^{2} \overline{\mathbf{z}}+\mathrm{O}(|\mathbf{z}|)^{4}, \quad z \in \mathbb{C}, \quad \alpha=\left(\alpha_{1}, \alpha_{2}\right) .
$$

A detailed derivation is given in the Appendix. In Section 2, we derive explicit formulas for the critical normal form coefficients up to cubic terms. Here we use an efficient method proposed by Iooss and coworkers [5] leading to compact formulas. Next, in Section 3, we propose a suitable new test-function to detect this bifurcation during continuation. This allows us to use these our normal form formulas in MATCONTM.

In section 4, we illustrate our new algorithms with a model from economy. Cournot introduced an economic theory for competition between a few large suppliers in 1838. Agiza [1] introduced and studied a Cournot triopoly model describing a Cournot game with three oligopolists. It describes the market share of three competitive companies producing homogeneous goods to be sold on a common market. The response incorporates a unimodal reaction 
function such that the model has $D_{3}$-symmetry. The map displays chaotic behaviour as studied in 1, 16] We introduce a small perturbation so that the input of one company is favoured over the other resulting in $\mathbb{Z}_{3}$-symmetry. Here we find a resonance $1: 1$ with $\mathbb{Z}_{3}$-symmetry, actually $D_{3}$-symmetry at the bifurcation, which is covered by our approach too. We analyze this bifurcation in detail. Finally, we discuss some future directions how other bifurcations with symmetry may be treated numerically.

\section{Center Manifold Reduction and Numerical Normal Forms}

Here we will derive the critical normal coefficients on the center manifold for (1.2). We will use a technique that combines center manifold reduction and normalization. For completeness we quickly review the method, and for more details we refer to [5, 11] in which the method was introduced, and [13, 15] where it was used for generic codim 2 bifurcations of maps.

We start with the $n$-dimensional system

$$
x \mapsto F(x), \quad F: \mathbb{R}^{n} \rightarrow \mathbb{R}^{n},
$$

at some critical value of the parameter $\alpha_{0}$, and therefore not indicated, and assume that $x_{0}=0$ is the non-hyperbolic fixed point. Furthermore, $A=F_{x}(0)$ has $m$-multipliers on the unit circle, corresponding to an $m$-dimensional center manifold. We assume that $F$ is sufficiently smooth, so that we can obtain the Taylor expansion

$$
F(x)=A x+\frac{1}{2 !} B(x, x)+\frac{1}{3 !} C(x, x, x)+\cdots
$$

where $B, C$ are multi-linear functions. We want to restrict system 2.1) to its $m$-dimensional center manifold that we parametrize by an $m$-dimensional variable $w$, i.e.

$$
x=H(w), \quad H: \mathbb{R}^{m} \rightarrow \mathbb{R}^{n} .
$$

On the center manifold the restricted map is given by the normal form $G$

$$
w \mapsto G(w) ; \quad G: \mathbb{R}^{m} \rightarrow \mathbb{R}^{m} .
$$

Note that we know the form of $G$, i.e. $(1.2)$, but not the values of the coefficients, and we need to find these values. To proceed, we use that the center manifold is invariant under the dynamics. This leads to the following homological equation relating the original system $F$ to the center manifold parametrized by $H$ and the normal form $G$ :

$$
F(H(w))=H(G(w)) \text {. }
$$


For the solution of the homological equation, we use power series. First, we write Taylor series for $G$ and $H$

$$
G(w)=\sum_{|\nu| \geq 1} \frac{1}{\nu !} g_{\nu} w^{\nu}, \quad H(w)=\sum_{|\nu| \geq 1} \frac{1}{\nu !} h_{\nu} w^{\nu},
$$

where $\nu=\left(\nu_{1}, \nu_{2}, \cdots, \nu_{m}\right)$ is a multi-index and $\nu !=\nu_{1} ! \nu_{2} ! \cdots \nu_{m} !$. Next, we collect the coefficients of $w^{\nu}$-terms in the homological equation. This gives a linear system for each $h_{\nu}$ of the form

$$
L_{\nu} h_{\nu}=R_{\nu}
$$

where, for each $\nu, L_{\nu}$ depends on $A$ and its multipliers, and $R_{\nu}$ contains known terms. Now, either $L_{\nu}$ is nonsingular and equation (2.5) has a solution, or $R_{\nu}$ satisfies the Fredholm solvability condition $\left\langle p, R_{\nu}\right\rangle=0$, where $p$ is any vector satisfying $\bar{L}_{\nu}^{T} p=0$. Typically, $L_{\nu}$ is singular when $R_{\nu}$ depends on the unknown coefficient $g_{\nu}$ of the normal form. In this way we get an expression for $g_{\nu}$. In some cases we need to find $h_{\nu}$ where $L_{\nu}$ is singular. Here, we use nonsingular bordered matrices, see also [9]. We consider the case that $L_{\nu}$ has a twodimensional null-space spanned by $q_{1}, q_{2}$. Similarly, let $\left\{p_{1}, p_{2}\right\}$ be a basis of the null-space of $L_{\nu}^{T}$. Then, we define the nonsingular $(n+2) \times(n+2)$-matrix

$$
\left(\begin{array}{ccc}
L_{\nu} & q_{1} & q_{2} \\
p_{1}{ }^{T} & 0 & 0 \\
p_{2}{ }^{T} & 0 & 0
\end{array}\right)
$$

Thus we are able to solve the nonsingular bordered system

$$
\left(\begin{array}{ccc}
L_{\nu} & q_{1} & q_{2} \\
p_{1}{ }^{T} & 0 & 0 \\
p_{2}{ }^{T} & 0 & 0
\end{array}\right)\left(\begin{array}{c}
h_{\nu} \\
s_{1} \\
s_{2}
\end{array}\right)=\left(\begin{array}{c}
R_{\nu} \\
0 \\
0
\end{array}\right)
$$

where $s_{1}, s_{2} \in \mathbb{R}$ are auxiliary variables. We can then find the solution $h_{\nu}=L_{\nu}^{i n v} R_{\nu}$, where $L_{\nu}^{i n v}$ denotes the generalized inverse. In addition, we have

$$
\left\langle p_{1}, h_{\nu}\right\rangle=\left\langle p_{2}, h_{\nu}\right\rangle=0 .
$$

In our case we have two multipliers equal to +1 and no other critical multipliers, so $m=2$. The truncated normal form, see Appendix, for this bifurcation is

$$
\left(\begin{array}{c}
\omega_{1} \\
\omega_{2}
\end{array}\right) \mapsto\left(\begin{array}{c}
\omega_{1}+\tilde{a} \omega_{1}^{2}-\tilde{a} \omega_{2}^{2}+c \omega_{1}^{3}+d \omega_{1}^{2} \omega_{2}+c \omega_{1} \omega_{2}^{2}+d \omega_{2}^{3} \\
\omega_{2}-2 \tilde{a} \omega_{1} \omega_{2}-d \omega_{1}^{3}+c \omega_{1}^{2} \omega_{2}-d \omega_{1} \omega_{2}^{2}+c \omega_{2}^{3}
\end{array}\right)
$$

where $\omega \in \mathbb{R}^{2}$. As the null-space of $A-I$ and $(A-I)^{T}$ is two-dimensional, we assume they are spanned by $\left\{q_{1}, q_{2}\right\}$ and $\left\{p_{1}, p_{2}\right\}$, respectively. In addition, we assume the following 
normalization $\left\langle q_{1}, q_{2}\right\rangle=\left\langle p_{1}, p_{2}\right\rangle=0,\left\langle p_{1}, q_{1}\right\rangle=\left\langle p_{2}, q_{2}\right\rangle=1$ and $\left\langle p_{1}, q_{2}\right\rangle=\left\langle p_{2}, q_{1}\right\rangle=0$. Collecting the linear terms in the homological equation, we obtain

$$
\begin{aligned}
& \omega_{1}: A h_{10}=h_{10}, \\
& \omega_{2}: A h_{01}=h_{01} .
\end{aligned}
$$

Therefore we choose $h_{10}=q_{1}$ and $h_{01}=q_{2}$.

\subsection{Formulas for the Critical Normal Form Coefficients}

The quadratic coefficient $\tilde{a}$ in $(2.7)$ is defined as $\tilde{a}=\operatorname{sign}(a) \sqrt{a^{2}+b^{2}}$, see Appendix. Therefore we must first compute $a$ and $b$ to compute $\tilde{a}$. Let us consider a general $\mathbb{Z}_{3}$-equivariant mapping up to order three,

$$
G\left(\omega_{1}, \omega_{2}\right)=\left(\begin{array}{c}
\omega_{1}+a \omega_{1}^{2}+2 b \omega_{1} \omega_{2}-a \omega_{2}^{2}+c \omega_{1}^{3}+d \omega_{1}^{2} \omega_{2}+c \omega_{1} \omega_{2}^{2}+d \omega_{2}^{3} \\
\omega_{2}+b \omega_{1}^{2}-2 a \omega_{1} \omega_{2}-b \omega_{2}^{2}-d \omega_{1}^{3}+c \omega_{1}^{2} \omega_{2}-d \omega_{1} \omega_{2}^{2}+c \omega_{2}^{3}
\end{array}\right)
$$

and set $G\left(\omega_{1}, \omega_{2}\right)=\left(g_{1}\left(\omega_{1}, \omega_{2}\right), g_{2}\left(\omega_{1}, \omega_{2}\right)\right)^{T}$ for the corresponding coordinate maps.

Collecting the $\omega^{2}$-terms from the homological equation, we obtain the following singular systems

$$
\begin{aligned}
\omega_{1}^{2}: & (A-I) h_{20}=-B\left(q_{1}, q_{1}\right)+a q_{1}+b q_{2}, \\
\omega_{1} \omega_{2}: & (A-I) h_{11}=-2 B\left(q_{1}, q_{2}\right)-2 a q_{2}+2 b q_{1}, \\
\omega_{2}^{2}: & (A-I) h_{02}=-B\left(q_{2}, q_{2}\right)-a q_{1}-b q_{2} .
\end{aligned}
$$

Since $(2.8)$ is $\mathbb{Z}_{3}$ - symmetric, we have

$$
\begin{aligned}
& \left\langle p_{1}, B\left(q_{1}, q_{1}\right)\right\rangle=-\left\langle p_{2}, B\left(q_{1}, q_{2}\right)\right\rangle=-\left\langle p_{1}, B\left(q_{2}, q_{2}\right)\right\rangle, \\
& \left\langle p_{2}, B\left(q_{1}, q_{1}\right)\right\rangle=\left\langle p_{1}, B\left(q_{1}, q_{2}\right)\right\rangle=-\left\langle p_{2}, B\left(q_{2}, q_{2}\right)\right\rangle .
\end{aligned}
$$

From $(2.9)$ we obtain,

$$
\begin{aligned}
a & =\left\langle p_{1}, B\left(q_{1}, q_{1}\right)\right\rangle, \\
b & =\left\langle p_{2}, B\left(q_{1}, q_{1}\right)\right\rangle, \\
h_{20} & =(A-I)^{i n v}\left(-B\left(q_{1}, q_{1}\right)+a q_{1}+b q_{2}\right), \\
h_{11} & =(A-I)^{i n v}\left(-2 B\left(q_{1}, q_{2}\right)+2 b q_{1}-2 a q_{2}\right), \\
h_{02} & =(A-I)^{i n v}\left(-B\left(q_{2}, q_{2}\right)-a q_{1}-b q_{2}\right),
\end{aligned}
$$

where according to (2.6) the vectors $h_{20}, h_{11}, h_{02}$ satisfy

$$
\left\langle p_{i}, h_{20}\right\rangle=0,\left\langle p_{i}, h_{11}\right\rangle=0,\left\langle p_{i}, h_{02}\right\rangle=0, \quad i=1,2 .
$$


At this point, we define new base vectors $v_{1}, v_{2}, w_{1}, w_{2}$ as follows with $\psi=\frac{1}{3} \tan ^{-1}\left(\frac{b}{a}\right)$ (see Appendix),

$$
\begin{gathered}
v_{1}=\cos (\psi) q_{1}+\sin (\psi) q_{2}, \quad v_{2}=-\sin (\psi) q_{1}+\cos (\psi) q_{2}, \\
w_{1}=\cos (\psi) p_{1}+\sin (\psi) p_{2}, \quad w_{2}=-\sin (\psi) p_{1}+\cos (\psi) p_{2}
\end{gathered}
$$

Under this choice, (2.8) is converted into the form as given in (2.7), where we use $c$ and $d$ again for the coefficients of the new cubic terms after rotation.

To compute $c$ and $d$, we consider (2.7) with $a=\tilde{a}$ and $b=0$. By collecting the $\omega^{3}$-terms in homological equation, we obtain the following singular systems

$$
\begin{aligned}
& \omega_{1}^{3}:(A-I) h_{30}=-2 B\left(v_{1}, h_{20}\right)-C\left(v_{1}, v_{1}, v_{1}\right)+c v_{1}-d v_{2}+2 \tilde{a} h_{20}, \\
& \omega_{1}^{2} \omega_{2}:(A-I) h_{21}=-2 B\left(v_{1}, h_{11}\right)-2 B\left(h_{20}, v_{2}\right)-3 C\left(v_{1}, v_{1}, v_{2}\right)+d v_{1}+c v_{2}-\tilde{a} h_{11}, \\
& \omega_{1} \omega_{2}^{2}:(A-I) h_{12}=-2 B\left(v_{1}, h_{02}\right)-2 B\left(h_{11}, v_{2}\right)-3 C\left(v_{1}, v_{2}, v_{2}\right)+c v_{1}-d v_{2}-2 \tilde{a} h_{20}-4 \tilde{a} h_{02}, \\
& \omega_{2}^{3}:(A-I) h_{03}=-2 B\left(v_{2}, h_{02}\right)-C\left(v_{2}, v_{2}, v_{2}\right)+d v_{1}+c v_{2}-\tilde{a} h_{11} .
\end{aligned}
$$

Since $(2.7)$ is $\mathbb{Z}_{3}-$ symmetric, we have

$$
\begin{aligned}
& \left\langle w_{1}, 2 B\left(v_{1}, h_{20}\right)+C\left(v_{1}, v_{1}, v_{1}\right)\right\rangle \\
& =\left\langle w_{2}, 2 B\left(v_{1}, h_{11}\right)+2 B\left(h_{20}, v_{2}\right)+3 C\left(v_{1}, v_{1}, v_{2}\right)\right\rangle \\
& =\left\langle w_{1}, 2 B\left(v_{1}, h_{02}\right)+2 B\left(h_{11}, v_{2}\right)+3 C\left(v_{1}, v_{2}, v_{2}\right)\right\rangle \\
& =\left\langle w_{2}, 2 B\left(v_{2}, h_{02}\right)+C\left(v_{2}, v_{2}, v_{2}\right)\right\rangle
\end{aligned}
$$

and

$$
\begin{aligned}
& -\left\langle w_{2}, 2 B\left(v_{1}, h_{20}\right)+C\left(v_{1}, v_{1}, v_{1}\right)\right\rangle \\
& =\left\langle w_{1}, 2 B\left(v_{1}, h_{11}\right)+2 B\left(h_{20}, v_{2}\right)+3 C\left(v_{1}, v_{1}, v_{2}\right)\right\rangle \\
& =-\left\langle w_{2}, 2 B\left(v_{1}, h_{02}\right)+2 B\left(h_{11}, v_{2}\right)+3 C\left(v_{1}, v_{2}, v_{2}\right)\right\rangle \\
& =\left\langle w_{1}, 2 B\left(v_{2}, h_{02}\right)+C\left(v_{2}, v_{2}, v_{2}\right)\right\rangle .
\end{aligned}
$$

From (2.10) we obtain,

$$
\begin{aligned}
& c=\left\langle w_{1}, 2 B\left(v_{1}, h_{20}\right)+C\left(v_{1}, v_{1}, v_{1}\right)\right\rangle, \\
& d=-\left\langle w_{2}, 2 B\left(v_{1}, h_{20}\right)+C\left(v_{1}, v_{1}, v_{1}\right)\right\rangle .
\end{aligned}
$$

Remark 2.1. Let us consider (1.1) with $\mathbb{D}_{3}$-symmetry, where $\mathbb{D}_{3}=\langle\{R, K\}\rangle$ with generators $R=R_{\frac{2 \pi}{3}}=\frac{1}{2}\left(\begin{array}{cc}-1 & -\sqrt{3} \\ \sqrt{3} & -1\end{array}\right)$ and $K=\frac{\sqrt{2}}{2}\left(\begin{array}{cc}-1 & 1 \\ 1 & 1\end{array}\right)$. This leads to a simpler critical 
normal form up to order three

$$
\left\{\begin{array}{l}
x_{1} \mapsto x_{1}+a x_{1}^{2}-a x_{2}^{2}+c x_{1}^{3}+c x_{1} x_{2}^{2} \\
x_{2} \mapsto x_{2}-2 a x_{1} x_{2}+c x_{1}^{2} x_{2}+c x_{2}^{3} .
\end{array}\right.
$$

We see that for $\mathbb{D}_{3}$-symmetry the coefficients $b$ and $d$ vanish generically. That is also the case in our example in section 4 .

\section{Bifurcation Analysis of Normal Form of Resonance 1 : 1 with $\mathbb{Z}_{3}$-Symmetry}

The fixed point persists at the bifurcation because of the symmetry. This implies that the fixed point does not disappear in a saddle-node bifurcation. Hence, the only possibility for a $1: 1$ resonance with $\mathbb{Z}_{3}$-symmetry to occur, is along a Neimark-Sacker (NS) bifurcation. So, for the bifurcation analysis of this codim 2 case, we briefly review the continuation of a Neimark-Sacker bifurcation in two parameters in MATCONTM. We then discuss how to adapt the detection and processing of this codim 2 point.

Numerical continuation exploits the implicit function theorem, where we have a defining system of $k$ equations in $k+1$ variables. The equations define the point of interest, here a Neimark-Sacker bifurcation, and the variables consist of the coordinates of the fixed point $x$, parameters $\alpha$ and an auxiliary variable $\kappa$. During continuation we compute a discrete sequence of points along the curve, and we use the tangent vector $v$ to predict the next point. A pseudo-arclength condition is added for Newton-corrections of the predicted point. Test functions $\varphi$ are used in MATCONTM to detect and locate codim 2 singularities along codim 1 bifurcation curves by monitoring sign changes and subsequently locating the bifurcation point more accurately.

For a Neimark-Sacker bifurcation, one can use the following minimally augmented defining system

$$
\left\{\begin{aligned}
f(x, \alpha)-x & =0, \\
s_{i_{1}, j_{1}}(x, \alpha, \kappa) & =0, \\
s_{i_{2}, j_{2}}(x, \alpha, \kappa) & =0,
\end{aligned}\right.
$$

consisting of $n+2$ equations for $n+3$ unknowns $x \in \mathbb{R}^{n}, \alpha \in \mathbb{R}^{2}, \kappa \in \mathbb{R}$. Here $\left(i_{1}, j_{1}, i_{2}, j_{2}\right) \in$ $\{1,2\}$ and $s_{i, j}$ are the components of $S$ :

$$
S=\left(\begin{array}{ll}
s_{11} & s_{12} \\
s_{21} & s_{22}
\end{array}\right)
$$

which is obtained by solving

$$
\left(\begin{array}{cc}
\left(f_{x}\right)^{2}(x, \alpha)-2 \kappa f_{x}(x, \alpha)+I_{n} & W_{b o r} \\
V_{b o r}^{T} & O
\end{array}\right)\left(\begin{array}{c}
V \\
S
\end{array}\right)=\left(\begin{array}{c}
0_{n, 2} \\
I_{2}
\end{array}\right),
$$


where $V_{\text {bor }}, W_{\text {bor }} \in \mathbb{R}^{n \times 2}$ are chosen so that the matrix in 3.2 is nonsingular. Only two linearly independent components of $S$ are used.

Along the Neimark-Sacker curve, $\kappa=\cos \theta$ denotes the real part of the critical multipliers $e^{ \pm i \theta}$. Note that a neutral saddle, a fixed point with two multipliers with product equal to 1 also satisfies 3.1 . This means there is a pair of multipliers $\gamma$ and $\frac{1}{\gamma}$ such that $\kappa=$ $\frac{1}{2}\left(\gamma+\frac{1}{\gamma}\right)>1$. In the generic case, the $1: 1$ resonance point marks the transition from a Neimark-Sacker bifurcation to a neutral saddle. Hence, in the generic case, the test function can be defined as $\varphi=\kappa-1$.

In the case of $\mathbb{Z}_{3}$-symmetry this generic test function is not suitable as $\kappa$ does not have a regular, but a quadratic zero along the Neimark-Sacker curve. Indeed, for the normal form (1.2) the multiplier of the trivial fixed point is given by $\lambda=1+\alpha_{1}+i \alpha_{2}$. The condition for a Neimark-Sacker bifurcation $|\lambda|=1$ then leads to $\alpha_{1} \approx-\frac{1}{2} \alpha_{2}^{2}$. So along the Neimark-Sacker bifurcation $\kappa=\cos \theta=1+\alpha_{1}$ increases and then decreases near the codim 2 point. Therefore, this quadratic zero can be detected as a change in sign in the component of the tangent vector corresponding to $\kappa$, this is the last component of the tangent vector $v$ and holds in general. Therefore, we define the test function $\varphi=v(e n d)$. Upon detection, we check $\kappa \approx 1$.

After detection, it is possible to adapt MATCONTM such that it evaluates the formulas for the critical normal form coefficients, and then the values of $\tilde{a}, c$ and $d$ in $(1.2)$ are reported. A precise list of changes and code can be found in [14], Supplement. Next, using the coefficients $\tilde{a}, c$, one can determine the Lyapunov coefficient $l_{1}=c-|\tilde{a}|^{2}$ indicating the stability of the invariant curve near this bifurcation. If $l_{1}<0$, then the invariant curve is stable.

\section{A Case Study in the Cournot Triopoly Game Model}

We introduce a three dimensional map with logistic reaction functions, positive adjustment coefficient $\lambda$ and positive parameter $\mu$ for measuring the intensity of the effect that one firm's actions has on the other firms. The model describes how the $i$ th firm adjusts its output $x_{i}(n), i=1,2,3$, for the next time step $x_{i}(n+1)$. The firm adjusts its production not immediately to the computed optimal production, but uses a weighted average of the previous and the newly determined optimal production with weights $1-\lambda$ and $\lambda$, respectively. In addition, we impose a slight asymmetry such that the influence of the neighbour in one direction is larger. The evolution of the outputs $x$ is given by

$$
\begin{aligned}
& x_{1}(n+1)=(1-\lambda) x_{1}(n)+\lambda \mu\left((1-\nu) x_{2}(n)\left(1-x_{2}(n)\right)+\nu x_{3}(n)\left(1-x_{3}(n)\right)\right), \\
& x_{2}(n+1)=(1-\lambda) x_{2}(n)+\lambda \mu\left((1-\nu) x_{3}(n)\left(1-x_{3}(n)\right)+\nu x_{1}(n)\left(1-x_{1}(n)\right)\right), \\
& x_{3}(n+1)=(1-\lambda) x_{3}(n)+\lambda \mu\left((1-\nu) x_{1}(n)\left(1-x_{1}(n)\right)+\nu x_{2}(n)\left(1-x_{2}(n)\right)\right),
\end{aligned}
$$


where we consider $0<\lambda, \nu<1$ and $\mu>0$. If $\nu=0.5$, system 4.1 has symmetry group $\mathbb{D}_{3}=\langle\{R, \hat{R}\}\rangle$ where $R=\left(\begin{array}{lll}0 & 0 & 1 \\ 1 & 0 & 0 \\ 0 & 1 & 0\end{array}\right)$ and $\hat{R}=\left(\begin{array}{lll}0 & 1 & 0 \\ 1 & 0 & 0 \\ 0 & 0 & 1\end{array}\right)$. If $\nu \neq 0.5$, system 4.1 has only symmetry group $\mathbb{Z}_{3}=\langle R\rangle$. The symmetric fixed points of 4.1) are the solutions to:

$$
\begin{aligned}
& x_{1}=x_{2}=x_{3}=x, \\
& x=(1-\lambda) x+\lambda \mu x(1-x) .
\end{aligned}
$$

We find that the triopoly model has two symmetric fixed points, $x_{*}=(0,0,0)$ and $x_{* *}=$ $\left(1-\frac{1}{\mu}, 1-\frac{1}{\mu}, 1-\frac{1}{\mu}\right)$. The fixed point $x_{* *}$ has multipliers

$$
\lambda_{1}=-\lambda \mu+\lambda+1, \quad \lambda_{2,3}=\left(\frac{1}{2} \lambda \mu-2 \lambda+1\right) \pm i \sqrt{3}\left(\lambda \mu \nu-\frac{1}{2} \lambda \mu-2 \lambda \nu+\lambda\right) .
$$

Setting the parameter $\lambda=0.4$, we find from the condition $\left|\lambda_{2}\right|=1$ the following NeimarkSacker bifurcation curve

$$
\mu=\frac{24 \nu^{2}-24 \nu+5+\sqrt{192 \nu^{2}-192 \nu+73}}{4\left(3 \nu^{2}-3 \nu+1\right)} .
$$

We now do a numerical continuation of (4.1). We fix $\lambda=0.4, \nu=0.75$ and vary $\mu$. Following the point $x_{* *}$, we encounter a supercritical Neimark-Sacker bifurcation for $x=(0.749071,0.749071,0.749071)$ and $\mu=3.985194$, as our analytical formula predicts. MATCONTM reports the following:

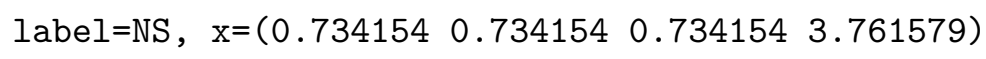

Next we select this NS point to start the continuation of a Neimark-Sacker bifurcation curve in two control parameters $\mu$ and $\nu$ and keeping $\lambda=0.4$ fixed. Using the adapted test-function during numerical continuation, MATCONTM reports the following:

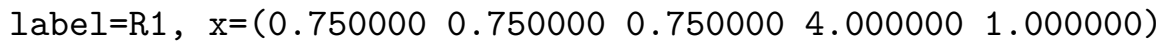

Normal form coefficient of R1:

$$
[\mathrm{aa}, \mathrm{c}, \mathrm{d}]=6.531973 \mathrm{e}-01,-2.844444 \mathrm{e}+00,-7.028934 \mathrm{e}-08
$$

We conclude that for $\mu=4$ and $\nu=0.5$, the fixed point $x=(0.75,0.75,0.75)$ has a double multiplier +1 . In addition, the normal form coefficients are reported using the new formulas. First, note that the coefficient $d$ is very small, close to numerical error as expected as at $\nu=0.5$ we have $\mathbb{D}_{3}$-symmetry. From $l_{1}=-1.992351$ we find that the Neimark-Sacker bifurcation in a neighbourhood of this point should be supercritical. To verify this, we have also computed 
Lyapunov exponents for parameters around the R1-Z3 point. We fixed $0.5 \leq c \leq 1$ as we would have obtained the same results for $c<0.5$ but then mirrored. Next, we then increased $b$ starting at $b=3$ until $b=5$. For each new parameter value of $b$, we used the final point of the previous orbit for the Lyapunov computation (if it was finite). The results are shown in Figure 1, with the continuation results and some bifurcation curves overlayed. The Neimark-Sacker bifurcation (NS) is indicated with a solid black line and the R1-Z3 point is indicated with a light-blue circle. The unfolding of this codim 2 point involves a heteroclinic bifurcation curve, indicated by a thick solid black line. Here, the invariant curve disappears through a heteroclinic connection of a secondary non- $\mathbb{Z}_{3}$-symmetric saddle fixed point. This saddle appears from a limit point curve $\left(\mathrm{LP}^{s}\right)$ together with a stable fixed point. Above the heteroclinic bifurcation, the orbit converges to this stable fixed point. Subsequently, this secondary stable fixed point undergoes a Neimark-Sacker bifurcation $\left(\mathrm{NS}^{s}\right)$. Further increasing $b$, the invariant curves evolve into chaotic attractors. This diagram indicates that several different attractors coexist, but we do not investigate that here.

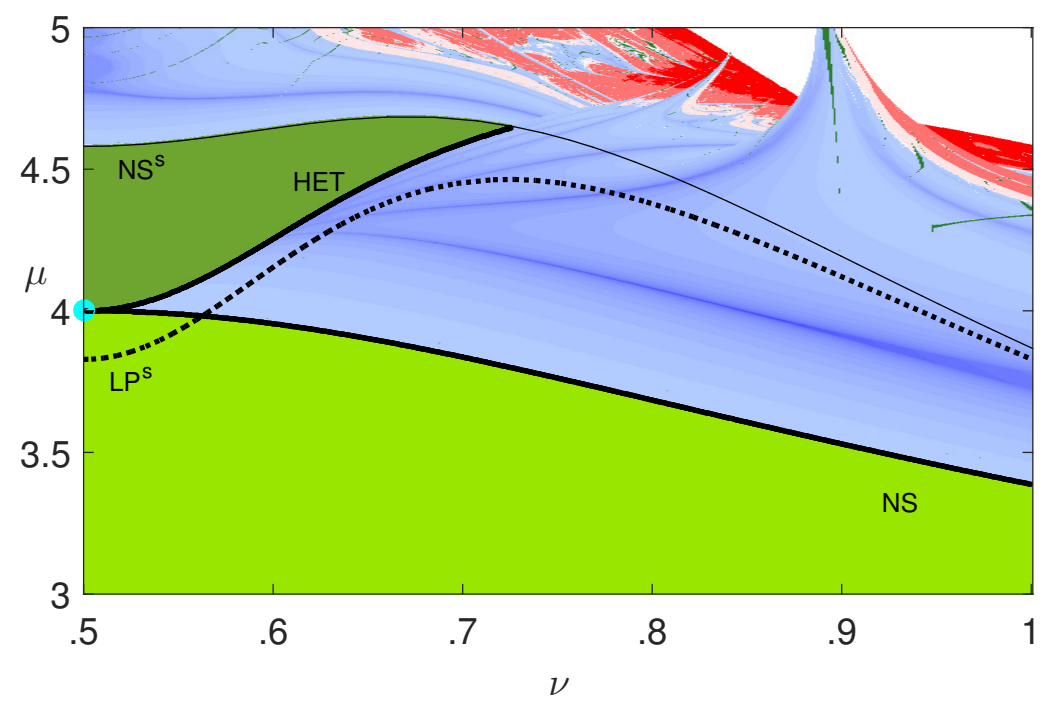

Figure 1: Bifurcation diagram of (4.1) around the R1-Z3 point (light blue) for $\lambda=0.4$. Black curves: Neimark-Sacker bifurcation of the symmetric (NS, thick solid) and secondary non-symmetrix $\left(\mathrm{NS}^{s}\right)$ fixed point (thin solid), Heteroclinic bifurcation of the non-symmetric saddle (HET, thick solid), limit point of the secondary non-symmetric saddle (LP ${ }^{s}$, thick dotted). The colors correspond to the observed attractors as follows: $\mathbb{Z}_{3}$-symmetric fixed point (light green, below NS), non-symmetric fixed point (green, between HET and $\mathrm{NS}^{s}$ ), cycles with higher period $(>7)$ (darkest green), invariant curve (blue with intensity scaled by second Lyapunov exponent), chaos (red with intensity scaled by first Lyapunov exponent), no attractor found (white). 


\section{Discussion}

We have shown that it is possible to modify the existing bifurcation toolbox MATCONTM to account for the presence of symmetries. For this we derived new formulas for the critical normal form coefficients and proposed a new test function. We illustrated the use of our methods using a triopoly model. We find excellent agreement between the bifurcation analysis and the observed dynamics, e.g. the negative Lyapunov coefficient and the stable invariant curve around the codim 2 point. Future work could consider other bifurcations with other symmetry groups. Our case study suggests how to derive appropriate test functions and normal form formulas for those cases. Here we relied on knowing the symmetry. A bigger challenge would be to automatically detect the correct symmetry. Another topic would be to consider the Equivariant Branching Lemma as it predicts the bifurcating solutions. This is theoretically well known, e.g. see the monograph [6]. For $\mathbb{Z}_{2}$-symmetry, e.g. a pitchfork bifurcation, one can determine the bifurcation equations [9], but it seems more involved for other symmetries to automate this.

\section{Appendix}

\section{Symmetry}

Since we want to obtain normal form of maps with $\mathbb{Z}_{3}$-symmetry, therefore we need the definitions of actions, representations and equivariance. For more details see [3, 7, 8].

Definition 6.1. Let $\Gamma$ be a Lie group and $V$ be a finite dimensional real vector space. We say that $\Gamma$ acts (linearly) on $V$, if there is a continuous mapping (the action)

$$
\begin{array}{r}
.: \Gamma \times V \mapsto V, \\
\quad(\gamma, v) \mapsto \gamma \cdot v,
\end{array}
$$

such that

(1) for each $\gamma \in \Gamma$, the mapping $\rho_{\gamma}: V \mapsto V$, defined by

$$
\rho_{\gamma}(v)=\gamma \cdot v
$$

is linear.

(2) if $\gamma_{1}, \gamma_{2} \in \Gamma$, then $\gamma_{1} \cdot\left(\gamma_{2} \cdot v\right)=\left(\gamma_{1} \gamma_{2}\right) \cdot v$.

The mapping $\rho$, that sends $\gamma$ to $\rho_{\gamma} \in G L(V)(G L(V)$ denotes the group of all invertible linear transformations of the vector space $V$ into itself) is then called a representation of $\Gamma$ on $V$. 
We consider the following representation $\mathbb{Z}_{3}$ on $\mathbb{R}^{2}$

$$
\text { 1. }\left(\begin{array}{l}
x_{1} \\
x_{2}
\end{array}\right)=\left(\begin{array}{l}
x_{1} \\
x_{2}
\end{array}\right), \frac{2 \pi}{3} \cdot\left(\begin{array}{c}
x_{1} \\
x_{2}
\end{array}\right)=R\left(\begin{array}{l}
x_{1} \\
x_{2}
\end{array}\right)
$$

where $\mathbb{Z}_{3}=\left\{1, \frac{2 \pi}{3}, \frac{4 \pi}{3}\right\}, R=R_{\frac{2 \pi}{3}}=\left(\begin{array}{cc}\frac{-1}{2} & \frac{-\sqrt{3}}{2} \\ \frac{\sqrt{3}}{2} & \frac{-1}{2}\end{array}\right)$.

We use this representation in our computations.

Definition 6.2. Let $\Gamma$ be a Lie group acting linearly on $V$. A mapping $F: V \rightarrow V$ commutes with $\Gamma$ or is $\Gamma$ - equivariant or has a symmetry group $\Gamma$, if $F(\gamma \cdot v)=\gamma \cdot F(v)$.

\section{Normal Form}

Let us consider system (1.1), suppose it has the fixed point $x_{0}=0$ at $\alpha=0$ with two multipliers +1 and other multipliers are not on unit circle and assume that system (1.1) has a symmetry group $\mathbb{Z}_{3}$, in other words $f(\gamma \cdot x, \alpha)=\gamma \cdot f(x, \alpha)$ where $\mathbb{Z}_{3}=\langle\gamma\rangle$. It is clear that the group action on the bifurcation parameters is the trivial action. The center manifold is two dimensional and restriction of (1.1) to a two dimensional center manifold has the following form

$$
x \mapsto L(\alpha) x+P(x ; \alpha), \quad x \in \mathbb{R}^{2}, \quad \alpha \in \mathbb{R}^{2},
$$

where $L(\alpha)$ is a matrix and $P$ is the nonlinear part of the map. Since $\mathbb{Z}_{3}$ is compact Lie group according to Ruelle's theorem ([12], chapter 7, section 4) the center manifold is invariant under $\mathbb{Z}_{3}$. So the center manifold reduction has the same symmetry. Therefore system 6.1) has a $\mathbb{Z}_{3}$-symmetry.

The equivariance of the map under $\mathbb{Z}_{3}$ implies that $L(\alpha)$ commutes with action of $\mathbb{Z}_{3}$ on $\mathbb{R}^{2}$, i.e. $L(\alpha) R=R L(\alpha)$. This fact and the assumption that $L(0)$ has a double multiplier +1 result in

$$
L(0)=\left(\begin{array}{ll}
1 & 0 \\
0 & 1
\end{array}\right)
$$

$L(\alpha)$ commutes with $R$, i.e. $R L(\alpha)=L(\alpha) R$, if we consider $L(\alpha)=\left(\begin{array}{cc}l_{11}(\alpha) & l_{12}(\alpha) \\ l_{21}(\alpha) & l_{22}(\alpha)\end{array}\right)$, from commuting $L(\alpha)$ with $R$ we obtain $l_{11}(\alpha)=l_{22}(\alpha)$ and $l_{12}(\alpha)=-l_{21}(\alpha)$. If we consider $L(\alpha)=\left(\begin{array}{cc}l_{11}(\alpha) & -l_{12}(\alpha) \\ l_{12}(\alpha) & l_{22}(\alpha)\end{array}\right)$, from $L(0)=I_{2 \times 2}$, we obtain that $l_{11}(0)=1$ and $l_{12}(0)=0$. We can consider $\mu_{1}=l_{11}(\alpha)-1$ and $\mu_{2}=l_{12}(\alpha)$, the map $\left(\alpha_{1}, \alpha_{2}\right) \mapsto\left(\mu_{1}, \mu_{2}\right)$ is regular at $\alpha=0$ provided $\frac{\partial l_{11}(\alpha)}{\partial \alpha_{1}} \frac{\partial l_{12}(\alpha)}{\partial \alpha_{2}}-\frac{\partial l_{11}(\alpha)}{\partial \alpha_{2}} \frac{\partial l_{12}(\alpha)}{\partial \alpha_{1}} \neq 0$, therefore we have $L(\mu)=\left(\begin{array}{cc}1+\mu_{1} & -\mu_{2} \\ \mu_{2} & 1+\mu_{1}\end{array}\right)$. 
According to [11, we can choose the normal form of the map such that $P$ satisfies

$$
\left\{\begin{array}{l}
P\left(L(0)^{\dagger} x ; \mu\right)=L(0)^{\dagger} P(x ; \mu) \\
P\left(R_{\frac{2 \pi}{3}}\left(\begin{array}{c}
x_{1} \\
x_{2}
\end{array}\right), \mu\right)=R_{\frac{2 \pi}{3}}\left(\begin{array}{c}
P_{1}(x, \mu) \\
P_{2}(x, \mu)
\end{array}\right) ;
\end{array}\right.
$$

where $L(0)^{\dagger}$ is the adjoint of $L(0), x=\left(x_{1}, x_{2}\right)^{T}, \mu=\left(\mu_{1}, \mu_{2}\right)^{T}$ and $P=\left(P_{1}, P_{2}\right)^{T}$. Therefore we have

$$
\left\{\begin{array}{l}
P_{1}\left(\frac{-1}{2} x_{1}-\frac{-\sqrt{3}}{2} x_{2}, \frac{\sqrt{3}}{2} x_{1}-\frac{1}{2} x_{2}, \mu\right)=-\frac{1}{2} P_{1}\left(x_{1}, x_{2}, \mu\right)-\frac{\sqrt{3}}{2} P_{2}\left(x_{1}, x_{2}, \mu\right), \\
P_{2}\left(\frac{-1}{2} x_{1}-\frac{-\sqrt{3}}{2} x_{2}, \frac{\sqrt{3}}{2} x_{1}-\frac{1}{2} x_{2}, \mu\right)=\frac{\sqrt{3}}{2} P_{1}\left(x_{1}, x_{2}, \mu\right)-\frac{1}{2} P_{2}\left(x_{1}, x_{2}, \mu\right) .
\end{array}\right.
$$

Expanding (6.4) up to third order in $x$, and setting each individual coefficient to zero in the expansion, we obtain

$$
\begin{gathered}
g_{00}=0, g_{02}=\frac{1}{2} h_{11}, g_{03}=-h_{30}, g_{11}=2 h_{20}, g_{12}=h_{21}, g_{20}=-\frac{1}{2} h_{11}, \\
g_{21}=-h_{30}, g_{30}=h_{21}, h_{00}=0, h_{02}=-h_{20}, h_{03}=h_{21}, h_{12}=h_{30},
\end{gathered}
$$

by choosing $a=g_{20}, b=\frac{1}{2} g_{11}, c=g_{30}$ and $d=g_{03}$ we have,

$$
\left\{\begin{array}{l}
P_{1}\left(x_{1}, x_{2} ; \mu\right)=a(\mu) x_{1}^{2}+b(\mu) x_{1} x_{2}-a(\mu) x_{2}^{2}+c(\mu) x_{1}^{3}+d(\mu) x_{1}^{2} x_{2}+c(\mu) x_{1} x_{2}^{2}+d(\mu) x_{2}^{3}, \\
P_{2}\left(x_{1}, x_{2} ; \mu\right)=\frac{1}{2} b(\mu) x_{1}^{2}-2 a(\mu) x_{1} x_{2}-\frac{1}{2} b(\mu) x_{2}^{2}-d(\alpha) x_{1}^{3}+c(\mu) x_{1}^{2} x_{2}-d(\mu) x_{1} x_{2}^{2}+c(\mu) x_{2}^{3},
\end{array}\right.
$$

By making the linear scaling $b \mapsto 2 b$ the normal form up to order three in $x$ is then,

$\left\{\begin{array}{l}x_{1} \mapsto\left(1+\mu_{1}\right) x_{1}-\mu_{2} x_{2}+a(\mu) x_{1}^{2}+2 b(\mu) x_{1} x_{2}-a(\mu) x_{2}^{2}+c(\mu) x_{1}^{3}+d(\mu) x_{1}^{2} x_{2}+c(\mu) x_{1} x_{2}^{2}+d(\mu) x_{2}^{3}, \\ x_{2} \mapsto \mu_{2} x_{1}+\left(1+\mu_{1}\right) x_{2}+b(\mu) x_{1}^{2}-2 a(\mu) x_{1} x_{2}-b(\mu) x_{2}^{2}-d(\mu) x_{1}^{3}+c(\mu) x_{1}^{2} x_{2}-d(\mu) x_{1} x_{2}^{2}+c(\mu) x_{2}^{3} .\end{array}\right.$

Introducing the complex coordinate $u=x_{1}+i x_{2}$, we can write the map as follows

$$
u \mapsto\left(\left(1+\mu_{1}\right)+i \mu_{2}\right) u+(a(\mu)+i b(\mu)) \bar{u}^{2}+(c(\mu)+i d(\mu)) u^{2} \bar{u} .
$$

We can simplify the quadratic part by a rotation $\mathbf{z}=e^{-i \psi} u$, where we set $\psi=\frac{1}{3} \tan ^{-1}\left(\frac{b}{a}\right)$. We then obtain

$$
\mathbf{z} \mapsto\left(\left(1+\mu_{1}\right)+i \mu_{2}\right) \mathbf{z}+\tilde{a}(\mu) \overline{\mathbf{z}}^{2}+(c(\mu)+i d(\mu)) \mathbf{z}^{2} \overline{\mathbf{z}}+\mathrm{O}(|\mathbf{z}|)^{4},
$$

where

$$
\tilde{a}=\operatorname{sign}(a) \sqrt{a^{2}+b^{2}} .
$$

Therefore, the normal form for a bifurcation with a double +1 multiplier and $\mathbb{Z}_{3}$ symmetry in real coordinates is as follows

$$
\left\{\begin{array}{l}
x_{1} \mapsto\left(1+\mu_{1}\right) x_{1}-\mu_{2} x_{2}+\tilde{a}(\mu) x_{1}^{2}-\tilde{a}(\mu) x_{2}^{2}+c(\mu) x_{1}^{3}+d(\mu) x_{1}^{2} x_{2}+c(\mu) x_{1} x_{2}^{2}+d(\mu) x_{2}^{3} \\
\left.x_{2} \mapsto \mu_{2} x_{1}+\left(1+\mu_{1}\right) x_{2}-2 \tilde{a}(\mu) x_{1} x_{2}-d(\mu) x_{1}^{3}+c \mu\right) x_{1}^{2} x_{2}-d(\mu) x_{1} x_{2}^{2}+c(\mu) x_{2}^{3}
\end{array}\right.
$$




\section{References}

[1] H.N. Agiza, G.I. Bischi and M. Kopel, Multistability in Dynamic Cournot game with three oligopolists, Math. Comput. Simul., 51, (1999), pp. 63-99.

[2] AUTO-07P : Continuation and Bifurcation Software for Ordinary Differential Equations, E.J. Doedel and B.E. Oldeman, Concordia University, Montreal, Canada, Available from https://sourceforge.net/projects/auto-07p/

[3] P. Chossat and M. Golubitsky, Iterates of maps with symmetry, SIAM J. Math. Anal. 19 (1988) pp. 1259-1270.

[4] A. Dhooge, W. Govaerts, Yu. A. Kuznetsov, H. G.E. Meijer \& B. Sautois, New features of the software MATCONT for bifurcation analysis of dynamical systems, Math. Comp. Mod. Dyn. Systems, 14 (2008), pp. 147-175.

[5] C. Elphick, E. Tirapegui, M.E. Brachet, P. Coullet and G. Iooss, A simple global characterization for normal forms of singular vector fields, Phys. D 29 (1987), pp. 95-127.

[6] M.J. Field. Dynamics and Symmetry, Advanced texts in mathematics, Vol. 3, Imperial College Press, London, 2007.

[7] M. Golubitsky and D.G. Schaeffer, Singularities and Groups in Bifurcation Theory, Vol. I, in: Appl. Math. Sci., vol. 51, Springer-Verlag, New York, 1985.

[8] M. Golubitkky, I. Stewart and D.G. Schaeffer, Singularities and Groups in Bifurcation Theory, Vol. II, in: Appl. Math. Sci., vol. 69, Springer-Verlag, New York, 1988.

[9] W. Govaerts, Numerical Methods for Bifurcations of Dynamical Equilibria, SIAM, Philadelphia, PA, 2000.

[10] W. Govaerts, R. Khoshsiar Ghaziani, Yu.A. Kuznetsov and H.G.E. Meijer. Numerical Methods for Two Parameter Local Bifurcation Analysis of Maps. SIAM. J. Sci. Comput., 29 (2007), pp. 2644-2667.

[11] G. Iooss and M. Adelmeyer, Topics in Bifurcation Theory and Applications, World Scientific, Singapore, 1999.

[12] Yu.A. Kuznetsov, Elements of Applied Bifurcation Theory, Springer-Verlag, Berlin, 1998, 3rd ed.

[13] Yu.A. Kuznetsov and H.G.E. Meijer, Numerical Normal Forms For Codim 2 Bifurcations of Fixed Points With at Most Two Critical Eigenvalues. SIAM. J. Sci. Comput., 26 (2005), pp. 1932-1954. 
[14] R. Mazrooei-Sebdani, Z. Eskandari and H.G.E. Meijer, Numerical and Theoretical Bifurcation Analysis of Double +1 Multiplier in $\mathbb{Z}_{3}$-Symmetric Maps, TW memorandum 2058, Department of Mathematics, University of Twente, available at http: //www.math.utwente.nl/publications/

[15] H.G.E. Meijer, Codimension 2 Bifurcations of Iterated Maps, PhD Thesis, Utrecht University, 2006.

[16] H. Richter and A. Stolk, Control of the Triple Chaotic Attractor in a Cournot Triopoly Model, Chaos, Solitons \& Fractals., 20 (2004), pp. 409-413. 